\title{
Heart Rate
}

National Cancer Institute

\section{Source}

National Cancer Institute. Heart Rate. NCI Thesaurus. Code C49677.

The number of heartbeats per unit of time, usually expressed as beats per minute. 